\title{
Glasgow supported self-management trial (GSuST) for patients with moderate to severe COPD: randomised controlled trial
}

\author{
(c) $\underset{10}{(1)(8)}$ OPEN ACCESS
}

\begin{abstract}
C E Bucknall consultant respiratory physician ${ }^{1}$, G Miller research fellow ${ }^{1}$, S M Lloyd biostatistician ${ }^{2}$, $\mathrm{J}$ Cleland professor of medical education ${ }^{3}$, S McCluskey senior research nurse ${ }^{1}$, M Cotton consultant respiratory physician ${ }^{1}, \mathrm{R}$ D Stevenson consultant respiratory physician ${ }^{1}, \mathrm{P}$ Cotton professor of learning and teaching ${ }^{4}$, A McConnachie deputy director ${ }^{2}$
\end{abstract}

${ }^{1}$ Department of Respiratory Medicine, Glasgow Royal Infirmary, Glasgow G4 OSF, UK; ${ }^{2}$ Robertson Centre for Biostatistics, University of Glasgow, Glasgow; ${ }^{3}$ Division of Medical and Dental Education, School of Medicine, University of Aberdeen, Aberdeen, UK; ${ }^{4}$ Undergraduate Medical School, University of Glasgow

\begin{abstract}
Objective To determine whether supported self management in chronic obstructive pulmonary disease (COPD) can reduce hospital readmissions in the United Kingdom.

Design Randomised controlled trial.

Setting Community based intervention in the west of Scotland.

Participants Patients admitted to hospital with acute exacerbation of COPD.

Intervention Participants in the intervention group were trained to detect and treat exacerbations promptly, with ongoing support for 12 months.

Main outcome measures The primary outcome was hospital readmissions and deaths due to COPD assessed by record linkage of Scottish Morbidity Records; health related quality of life measures were secondary outcomes.

Results 464 patients were randomised, stratified by age, sex, per cent predicted forced expiratory volume in 1 second, recent pulmonary rehabilitation attendance, smoking status, deprivation category of area of residence, and previous COPD admissions. No difference was found in COPD admissions or death (111/232 (48\%) v 108/232 (47\%); hazard ratio $1.05,95 \%$ confidence interval 0.80 to 1.38$)$. Return of health related quality of life questionnaires was poor $(n=265 ; 57 \%)$, so that no useful conclusions could be made from these data. Pre-planned subgroup analysis showed no differential benefit in the primary outcome relating to disease severity or demographic variables. In an exploratory analysis, $42 \%(75 / 150)$ of patients in the intervention group were classified as successful self managers at study exit, from review of appropriateness
\end{abstract}

of use of self management therapy. Predictors of successful self management on stepwise regression were younger age $(P=0.012)$ and living with others $(P=0.010)$. COPD readmissions/deaths were reduced in successful self managers compared with unsuccessful self managers (20/75 (27\%) v51/105 (49\%); hazard ratio $0.44,0.25$ to $0.76 ; P=0.003)$.

Conclusion Supported self management had no effect on time to first readmission or death with COPD. Exploratory subgroup analysis identified a minority of participants who learnt to self manage; this group had a significantly reduced risk of COPD readmission, were younger, and were more likely to be living with others.

Trial registration Clinical trials NCT 00706303.

\section{Introduction}

Self management has a well established evidence base for asthma and has been actively investigated as a useful strategy for patients with chronic obstructive pulmonary disease (COPD), with a growing evidence base for beneficial and unhelpful practices. ${ }^{1-3}$ It aims to develop patients' coping skills to maintain as active a lifestyle as possible, promote correct use of drugs, and encourage the early identification of increasing symptoms heralding an exacerbation, so that these can be treated early. Early treatment of exacerbations has been shown to reduce morbidity and effect on quality of life. ${ }^{4}$ Case management is a related technique for the support of patients with chronic disease, concentrating on the provision of support by health professionals so that patients can obtain prompt and appropriate access to care for their health related problems. This approach has been shown 
to be beneficial in chronic heart failure but not definitively in COPD. ${ }^{5}$

At the time this study started, the evidence base for self management for COPD patients was weak. ${ }^{6}$ More recent studies, which have mostly used a combined approach involving elements of both self management training and case management, have been more robust and shown benefits. ${ }^{2}$ No randomised controlled studies have been done in the UK healthcare system.

Acute exacerbations of COPD are the most common cause of emergency respiratory admission. ${ }^{7}$ The 2008 national COPD audit reported a 90 day readmission rate of $34 \% .^{8}$ This imposes a considerable burden on patients and on the health service, in terms of workload and cost. ${ }^{49}$ This study therefore set out to test whether combined self management and case management (supported self management) could reduce readmissions to hospital in patients with COPD who had been admitted with acute exacerbations of COPD in the west of Scotland.

\section{Methods}

\section{Participant recruitment and randomisation}

Eligible patients were those with COPD who had been admitted to hospital with an acute exacerbation of COPD. We defined COPD as chronic irreversible airflow limitation with forced expiratory volume in one second $\left(\mathrm{FEV}_{1}\right)$ less than $70 \%$ predicted and $\mathrm{FEV}$ //forced vital capacity ratio of less than $70 \%$. Forced vital capacity is defined as the total amount of air that can be expelled from the chest by a forced expiratory manoeuvre. We excluded patients with a history of asthma or left ventricular failure, which might cause confusion about the cause of breathlessness; evidence of active malignant disease, which could influence mortality; or any evidence of confusion/poor memory, assessed with the abbreviated mental test (scores of $9 / 10$ or $10 / 10$ required). ${ }^{10}$ We identified potentially eligible patients during or shortly after their hospital admission in all six acute Glasgow hospitals and contributing Lanarkshire hospitals to which eligible patients with a Glasgow postcode were admitted. We augmented this by reviewing patients attending pulmonary rehabilitation and crosschecking for evidence of hospital admission.

We gave or sent potentially eligible patients a study information leaflet and then contacted them, usually by telephone, to establish their interest in participation. We made a home visit to check eligibility and to enrol and subsequently randomise the patient, if appropriate.

We used a minimisation technique to stratify randomisation of participants by demographic factors (deprivation category of area of residence, ${ }^{11}$ age and sex, $\mathrm{FEV}_{1}$ per cent predicted at the time of randomisation, smoking status, participation in pulmonary rehabilitation within two years, and number of previous admissions) to control for key aspects of disease severity and predictors of readmission. We constructed a computer generated sequence by using the method of randomised permuted blocks of length four, with two allocations being made at random and two by minimisation. Treatment group allocations were obtained by telephone, after baseline assessments had been made. This registered the participant on the system, and a researcher entered the characteristics necessary for the minimisation algorithm by using an interactive voice response system. The researcher did not know whether a participant was being allocated at random or by minimisation and could therefore not determine the next treatment allocation before enrolling each participant.

\section{Study protocol}

All participants had their long term treatment optimised and inhaler technique checked and were offered appropriate smoking cessation advice and pulmonary rehabilitation if this had not been done within the previous two years. ${ }^{12}$ Participants received monthly telephone calls from an independent researcher, blinded to the patients' randomisation status, to collect information on health service usage and exacerbations. They were asked to complete daily diary cards and the questionnaires described below at six and 12 months and reminded to complete these at the time of the appropriate monthly telephone call and return them by prepaid postage. Diary cards were based on those of Woolhouse et al (box 1). ${ }^{13}$

Participants in the intervention group received supported self management. Study nurses' training was based on self regulation theory (box 2; further details available from corresponding author). ${ }^{14}$ The intervention aimed to empower patients to manage their COPD independently by improving their understanding of disease and monitoring of symptoms and by developing their confidence to carry out appropriate actions, such as altering treatment early in the evolution of an exacerbation or initiating contact with their usual medical attendant. Self management materials based on the Living Well with COPD programme and previously adapted for the UK population and healthcare setting by an iterative process, were used (Sue Mason, personal communication). ${ }^{15}$ Web appendix A shows an example.

Participants received four 40 minute individual training sessions at home from a study nurse, fortnightly over a two month period, with further home visits at least every six weeks (but more frequently on request) thereafter for a total of 12 months. Nurses showed patients how to use their symptom diary cards to recognise deteriorating symptoms and activate their self management plan. Follow-up visits were patient centred, based on individual needs as well as reviewing and reinforcing basic self management messages on the basis of diary card content.

We used Anthonisen's criteria for characterisation of acute exacerbations of COPD,${ }^{16}$ defining an exacerbation as a 2 point change from the participant's normal level of breathlessness and sputum colour and quantity or the development of new upper airway symptoms for two consecutive days. We defined the end of an exacerbation as a return of respiratory symptoms to baseline for three days. Participants were trained to identify and treat exacerbations associated with a 2 point deterioration in sputum colour with antibiotic (amoxicillin $500 \mathrm{mg}$ three times daily or oxytetracycline $500 \mathrm{mg}$ four times daily for seven days) and those associated with increased breathlessness, mucoid sputum, or upper airway symptoms with prednisolone $25 \mathrm{mg}$ for five days. This dose was a compromise between the convenience of the $25 \mathrm{mg}$ tablet and the higher doses used for established exacerbations, ${ }^{12}$ together with the use of treatment earlier in an exacerbation, taking account of the possibility that participants would overuse treatment with its associated side effects.

The control group continued to be managed by their general practitioner, hospital based specialists, or both (including the use of emergency out of hours services and the NHS 24 hour helpline).

\section{Outcome measures}

The primary end point was the time to first acute hospital admission with an exacerbation of COPD or death due to COPD within 12 months of randomisation. The participant's usual medical attendant decided on the need for hospital admission, independently of the study team. The Information and Statistics 


\section{Box 1: Diary card assessment of symptoms}

Breathing (1=better than usual, 2=normal/usual, $3=$ =worse than usual, $4=$ much worse than usual Colour of sputum (using chart with five grades of colour from $1=$ mucoid to $5=$ very purulent (deep green)) Amount of sputum produced ( $0=$ none, $1=$ some, up to teaspoonful, $2=a$ little (tablespoon), $3=$ moderate (eggcup or more), $4=a$ lot (cupful or more)

Type of sputum (1=watery, $2=$ sticky liquid, $3=$ semisolid, $4=$ solid)

Associated symptoms: general wellbeing (1-4), cough (0-3), chest pain (yes/no), cold or flu symptoms (yes/no)

Nocturnal symptoms $(0=$ slept well, $1=$ woke once because of chest symptoms, $2=$ woke twice because of chest symptoms, $3=$ woke more than twice because of chest symptoms, $5=$ unable to sleep because of symptoms)

\footnotetext{
Box 2: Training programme monitoring strategy

Nurses were trained to deliver a structured self management programme in four fortnightly home visits, each lasting about 40 minutes. During home visits, the nurses discussed:

- The diagnosis of chronic obstructive pulmonary disease (COPD)

- The events that led up to the patient being admitted to hospital

- The nature of exacerbations of COPD, particularly the concept of infective and non-infective exacerbations

- How to recognise the early signs of a developing exacerbation

- How to manage infective and non-infective exacerbations in future, using the diary card to monitor levels of breathlessness and sputum colour

- How drugs work for the long term treatment and acute exacerbations.

During follow-up visits, in addition to reviewing the above categories, nurses reviewed the patient's experiences since last seen and used them as an opportunity to reinforce appropriate self management behaviour and influence unhelpful coping strategies. Information booklets about COPD $(n=6)$ were left with the patient, together with a contact telephone number for advice during working hours.

Nurses without previous respiratory training completed three half day training sessions, with an equal emphasis on:

- Aspects of COPD pathophysiology and pharmacology

- Communications strategies designed to empower patients (including reinforcing effective strategies, active listening, responding to patients' concerns, maintaining interactive conversation).

In addition, observed home interviews took place with one trainer $(\mathrm{JC})$ who used a checklist to record communication behaviours and provide feedback. $^{26}$

Quality assurance of home visits during study

At regular intervals throughout the study, the senior nurse (SL) did paired home visits with study nurses, observing interactions with patients and scoring these to provide feedback on performance.
}

Division of the NHS in Scotland independently collated record linked data on all hospital admissions and deaths for the 12 month period after randomisation for all participants.

Secondary outcome measures were change from baseline at six and 12 months in St George's respiratory questionnaire (SGRQ) ${ }^{17}$ in which higher scores indicate greater morbidity; hospital anxiety and depression scale (HADS), ${ }^{18}$ in which higher scores indicate greater likelihood of anxiety or depression; COPD self-efficacy scale (CSES),${ }^{19}$ in which higher scores indicate a greater sense of self efficacy; and EuroQol 5D, a generic quality of life questionnaire, ${ }^{20}$ in which larger areas under the curve indicate better quality of life.

Planned subgroup analyses of differences in the primary end point between groups defined by the minimisation variables described earlier aimed to assess the possible effect of these factors on outcomes. Within the first few months, we realised that some participants accepted self management more readily than others. Therefore, as participants in the intervention group completed their 12 month period of follow up, we classified them as either a "successful self manager" or not after case based review by the study team (respiratory specialist, research registrar, and study nurses) of whether the diary card record showed a two point deterioration for two consecutive days before prednisolone, antibiotics, or both were started (assessed by detailed scrutiny of each patient's diary card data) and additional scrutiny of study nurses' contemporaneous clinical notes of each patient's handling of exacerbations. The study team were blind to information on hospital admissions when these classifications were made. These were collated electronically at the end of the study.

\section{Statistical analysis, study numbers, and power calculation}

To achieve $90 \%$ power to detect a reduction in the composite end point of COPD related readmission or death rate from $40 \%$ to $25 \%$, we needed 216 patients per group on the basis of a continuity corrected $\chi^{2}$ test for association. We aimed to recruit 250 patients per group, to allow for $13.5 \%$ attrition.

Data for the primary outcome (time to first acute COPD hospital admission or death) were available for all patients and analysed with a Cox proportional hazards model adjusting for the stratification variables. We present results as hazard ratios (intervention:control) with corresponding 95\% confidence intervals and $\mathrm{P}$ values. We considered $\mathrm{P}$ values less than 0.05 to be statistically significant. We produced Kaplan-Meier plots to compare the unadjusted survival rates between the groups.

We used analysis of covariance models to analyse the secondary outcomes (change from baseline in questionnaire scores at 6 and 12 months), adjusting the models for the baseline score and stratification variables. We present results as estimated differences (intervention minus control) with $95 \%$ confidence intervals and $\mathrm{P}$ values.

We used Cox proportional hazards models to investigate differences in the effect of the intervention between subgroups as determined by the stratification variables by including a "intervention by subgroup" interaction term in the model. We present the hazard ratios and $95 \%$ confidence intervals for the intervention effects within each of the subgroups, along with the $\mathrm{P}$ values for the intervention by subgroup interaction.

We identified predictors of successful self management initially from univariate logistic regression models. We then investigated 
multivariately those variables identified as significant at the univariate level. A backwards stepwise selection method identified those variables that were independently associated with successful self management. Within the intervention arm of the study, we compared successful and unsuccessful self managers in terms of the study outcomes, using the methods detailed above.

\section{Results}

Between June 2007 and May 20091405 patients were screened, and, of 995 eligible cases, 464 (47\%) were randomised to the study (fig $1 \Downarrow$ ). Median time to randomisation from the time of discharge from hospital for the index exacerbation was 29 days, with no difference between groups. Intervention and control groups were well matched for demographic and other variables (table $1 \Downarrow$ ). Fifty-three $(11 \%)$ patients formally withdrew during the study, which was completed in May 2010.

\section{Primary end point}

One hundred and eleven (48\%) intervention participants and $108(47 \%)$ control participants were readmitted to hospital with COPD or died of COPD within 12 months. We found no significant difference between groups in terms of the risk of hospital admission or death due to COPD (hazard ratio 1.05, $95 \%$ confidence interval 0.80 to $1.38 ; \mathrm{P}=0.73$ ). In total, 39 deaths from COPD and 52 deaths from all causes occurred, with no significant differences in terms of risk between the groups (table $2 \Downarrow$; fig $2 \Downarrow$ ). The pre-planned subgroup analyses also found no evidence of intervention effects in any subgroup of the study population (fig $3 \Downarrow$ ).

\section{Secondary end points}

Baseline questionnaires and at least one follow-up questionnaire were available for $265(61 \%)$ of the 433 patients still participating in the study at the six month time point. Participants who failed to return questionnaires were more likely to be female, to be current smokers, to be living in areas of greatest deprivation, to have been allocated to the control group, or to have had a hospital admission due to COPD in the previous 12 months. They were also less likely to have attended pulmonary rehabilitation in the two years before randomisation. Those who failed to return follow-up questionnaires had higher baseline St George's respiratory questionnaire impact sub-scores, higher hospital anxiety and depression scale scores, and lower COPD self efficacy scores, indicative of greater morbidity and less sense of self efficacy (web appendix B).

Of the 265 participants who returned baseline questionnaire and at least one of the follow-up questionnaires, 150/232 (65\%) were from the intervention group and 115/232 (50\%) were from the control group. The number of questionnaires available for analysis varied between outcomes and time points owing to the number of questionnaires returned and the completeness of the returned questionnaires. Despite the lack of effect on St George's respiratory questionnaire symptom and activity sub-scores, we saw a marked improvement in impacts sub-score in the intervention group, contributing to a borderline significant effect of intervention on total St George's respiratory questionnaire score at 12 months (table $2 \Downarrow$ ). Hospital anxiety and depression scale anxiety score was also significantly improved at 12 months, although we found no significant differences in hospital anxiety and depression scale depression scores or COPD self efficacy scores between the groups (table $2 \Downarrow$ ).

\section{Successful self management}

Seventy-five (42\%) of 180 intervention group participants who completed 12 months' follow-up were judged to have become successful self managers; this group were younger, had lower baseline COPD self efficacy scores (indicating lower self efficacy), and were more likely to be living with others. Apart from this, the groups were well matched for demographic and disease severity variables (table $3 \Downarrow$ ). The independent predictors of successful self management identified from stepwise modelling were younger age $(\mathrm{P}=0.012)$ and living with others $(\mathrm{P}=0.010)$ (table $4 \Downarrow)$.

We found a significant reduction in readmissions among successful self managers, compared with those in the intervention group who did not master the technique $(27 \% \mathrm{v}$ $49 \%$; hazard ratio $0.44,0.25$ to 0.76 ; $\mathrm{P}=0.003$ ) (table $5 \Downarrow$; fig $4 \Downarrow)$. This was associated with a difference in the time to first exacerbation in favour of the successful group. We found no significant difference in terms of all cause hospital admissions (table $5 \Downarrow$ ).

We also compared the primary end point between successful self managers and control group participants who completed 12 months of follow-up because, although in the intention to treat analysis participants in the control group had a readmission rate of $47 \%$ (table $2 \Downarrow$ ), this rate was $43 \%$ (75/174) for those who completed the 12 months' follow-up. These rates are similar to those for the unsuccessful self managers. Comparison of the primary end point of successful self managers and control group participants completing the 12 month follow-up showed a smaller but still significant benefit (hazard ratio 0.60, 0.36 to 0.99; $\mathrm{P}=0.045$ ). Web appendix $\mathrm{D}$ shows the de novo analysis of the primary end point for successful and unsuccessful self managers with correction for possible confounding by all stratification variables, COPD self efficacy score at baseline and whether participants were living alone or with others; this shows that the difference remained significant $(\mathrm{P}=0.03)$.

\section{Discussion}

In this study in the UK healthcare setting, supported self management had no effect on readmissions or deaths due to COPD and planned subgroup analyses showed no evidence of a differential effect on outcome in relation to factors associated with disease severity $\left(\mathrm{FEV}_{1}\right.$ per cent predicted at the time of randomisation, smoking status, number of previous admissions), demographics (deprivation category of area of residence, age, sex), or attendance at pulmonary rehabilitation within two years. Although not pre-specified, we realised within the first six months of running the intervention that some patients were more receptive to the concept of early recognition and treatment of exacerbations. This allowed the study to be adapted so that patients in the intervention group were classified at study exit as successful self managers or not, by assessment of the appropriateness of their use of treatment from review of diary card records of symptoms in the days before they started treatment. This classification was made independently of information on readmissions to hospital. Exploratory analyses showed that successful self managers had a significant reduction in the primary end point, and multivariate analysis showed that successful self managers were more likely to be younger and living with others; other possible confounders including smoking status were not significantly different (table $3 \Downarrow$, web appendix D). Successful self managers had significantly lower/worse self efficacy scores at baseline, although this difference did not survive multivariate analysis, suggesting that it was related to the other variables. 


\section{Strengths and weaknesses of study}

The robust study design, the large cohort that allowed subgroup analyses, and the use of record linked data from all hospital admissions in Scotland for all randomised cases are important positive features of this study. The low participation rate (47\%) theoretically limits the generalisability of the findings. However, given the commitment needed from patients to actively monitor their condition and adjust treatment within the study, patients not agreeing to participate in a trial of this design would probably not be more likely to benefit than those studied. Web appendix $\mathrm{C}$ shows the limited comparison possible on demographic data of patients who participated and those who declined, showing that participants were slightly younger on average (mean age $69 v 70$ years; $\mathrm{P}=0.07$ ) and were less likely to be living in the most highly deprived areas of residence; despite this, $61 \%$ of the study population came from the most deprived areas $(61 \%$ in deprivation categories 6 and 7, compared with $69 \%$ of non-participants; $\mathrm{P}=0.02$ ). Nevertheless, given that the primary analysis was negative and none of the pre-planned subgroup analyses found any evidence of benefit,

non-participation is unlikely to have biased this result. Our recruitment rate, at $47 \%$ of eligible patients, was similar to that reported in previous studies (for example, $41 \%$ in Bourbeau et al, ${ }^{15} 49 \%$ in Rice et $\mathrm{al}^{21}$ ), so that this problem applies to the entire literature, an aspect of methodology not covered in the recent Cochrane review. ${ }^{2}$ The findings of our study in terms of participation rates and lack of effect on important outcomes highlight the fact that this practice cannot be recommended for the generality of COPD cases of this level of severity in the UK healthcare setting.

Completion rates for study questionnaires were also disappointing and were lower in the control arm of the study. Consequently, the apparent improvements in the intervention arm (impacts subscale of St George's respiratory questionnaire, hospital anxiety and depression scale anxiety) could be biased, and these results cannot be taken as convincing evidence in favour of the intervention.

\section{Differences between this and other studies}

This study has not shown an effect on readmissions to hospital with supported self management, at variance with the conclusion of the recent Cochrane review, ${ }^{2}$ which included several smaller negative studies. Our study was adequately powered for the primary end point, and the study population was very similar to those of previously reported positive studies in terms of reported $\mathrm{FEV}_{1}$ and other disease related variables. ${ }^{15}{ }^{21}$ Our control and intervention groups were well matched in terms of important variables such as female sex,${ }^{22}$ depression, ${ }^{23}$ and living alone. ${ }^{24}$ Comparing socioeconomic deprivation between studies in different countries is difficult, but our intervention was applied to a representative group of patients living in highly deprived areas where COPD is known to be concentrated. ${ }^{89}$ Several possible reasons could explain why this study did not reproduce the whole group benefits seen in other recent studies: differences in interventions and reporting of outcomes, in healthcare systems, in the eligibility of patients for studies, and in the effectiveness of the intervention itself. Rice et al had a combined end point of emergency department visit or readmission to hospital, with no significant benefit in terms of readmission alone. ${ }^{21}$ In the UK, the equivalents to emergency departments are accident and emergency and out of hours services; our participants reported low usage of these services-a median of 0 (range $0-3$ ) visits to accident and emergency and $0(0-8)$ out of hours contacts over the 12 month follow-up period, with no differences between groups. In terms of intervention differences, Bourbeau et al's intervention included an exercise component, ${ }^{15}$ whereas we used stratification on the basis of recent attendance at pulmonary rehabilitation to control for the known beneficial effects of such rehabilitation. ${ }^{25}$ The effect of differences in healthcare systems is beyond the scope of this paper, but factors such as payment structures, incentives, and the characteristics of patients who are eligible for such interventions in different healthcare systems may well be important. The observation from this study that an intervention which has been shown to be effective in other settings has not produced the same benefits in the UK underscores the possible effect of such factors.

With regard to the efficacy of the intervention reported here, training was well structured (box 2), with didactic teaching, interactive exercises, and discussion, coupled with feedback on use of skills in a real life interaction by one trainer (JC) using a structured method of feedback. ${ }^{26}{ }^{27}$ All study nurses were assessed as competent before they were allocated patients to support, and the observation that the intervention produced an effect in a substantial subgroup of patients argues for its efficacy.

\section{What this study adds}

This study clearly casts doubt on the effectiveness of the wholesale introduction of supported self management in the UK healthcare setting. It provides important subgroup analyses that show no difference in effect of the intervention in relation to previously recognised factors associated with disease severity or with demographic variables. The finding that only $42 \%$ of intervention group patients learnt to self manage, and that this subgroup had a significant reduction in the primary end point, extends our current understanding of the possible effect of supported self management. Bischoff et al reported a similar level of effective use of a self management plan in a nested study of exacerbations occurring during a rehabilitation trial in Canada, ${ }^{28}$ where younger age, coexisting cardiac disease, and uptake of influenza vaccination were the independent predictors of adherence to self management plans. Together, these studies begin the process of defining the characteristics of patients who may benefit from training and support in self management.

\section{Unanswered questions and future research}

Our findings show that supported self management cannot be recommended for the generality of COPD patients treated for exacerbated symptoms in the UK setting. The finding that a significant minority of patients do derive benefit from this intervention must be regarded as hypothesis generating, and further investigation of the characteristics of successful self managers is needed. Understanding this will help clinicians to target limited resources efficiently and effectively, and, by exploring the nature of this population further, we may be able to identify how best to support those patients who do not benefit from self management. Further study of the characteristics of successful self managers should allow predictive tools for this and for the objective measurement of efficacy to be created.

\section{Conclusions}

This study found that supported self management had no effect on readmissions to hospital or death due to COPD in a large representative group of patients in the west of Scotland. Subgroup analysis showed no differential effect in relation to known factors of disease severity or demographics. Exploratory analyses showed that only $42 \%$ of the intervention group were classified as successful self managers at the end of the study period (12 months) on the basis of their recognition and 
appropriate use of treatment, and this subgroup had a significantly reduced risk of readmission or death. Predictors of successful self management are now emerging from this and other studies, and this analysis shows that younger patients and those not living alone are more likely to use self management techniques and derive benefit from them.

Contributors: CEB, GM, MC, RDS, JC, PC, and AMC were involved in study conception. CEB, SM, and GM were involved in design and management of the study. CEB, JC, PC, AMC, and SL were involved in drafting and revising the manuscript. All authors were involved in analysis and interpretation of data and final approval of the version to be published. CEB is the guarantor.

Funding: In addition to funding from the Chief Scientist Office, Scottish Health Department (CZH/4/246), this study was supported by educational grants from Boehringer Ingelheim, GlaxoSmithKline, and Astra Zeneca. The commercial sponsors played no part in the design, implementation, or analysis of results from this trial or in the preparation of this paper. Competing interests: All authors have completed the ICMJE uniform disclosure form at www.icmje.org/coi_disclosure.pdf (available on request from the corresponding author) and declare: in addition to the Chief Scientist Office grant (CZH/4/246), CEB's institution received financial support for the employment of a research fellow from Boehringer Ingelheim, GlaxoSmithKline, and Astra Zeneca, and JC holds other grants; no financial relationships with any organisations that might have an interest in the submitted work in the previous three years; no other relationships or activities that could appear to have influenced the submitted work.

Ethical approval: The original study and subsequent amendments to the protocol were approved by the Primary Care Ethics Subcommittee, Greater Glasgow and Clyde NHS Board.

Data sharing: The dataset is still subject to further analyses but will continue to be held by the Robertson Centre for Biostatistics; contact the corresponding author for further information.

1 Gibson PG, Powell H, Wilson A, Abramson MJ, Haywood P, Bauman A, et al Self-management education and regular practitioner review for adults with asthma. Cochrane Database Syst Rev 2002;3:CD001117.

2 Effing T, Monninkhof EEM, van der Valk P, van der Palen J, van Herwaarden CL, Partridge MR, et al. Self management education for patients with COPD. Cochrane Library, 2009.

3 Walters JAE, Turnock AC, Walters EH, Wood Baker R. Action plans and limited patient education only for exacerbations of COPD (review). Cochrane Database Syst Rev 2010:5:CD005074.

4 Wilkinson TMA, Donaldson GA, Hurst JR, Seemungal TAR, Wedzicha JA. Early therapy improves outcomes of exacerbations of chronic obstructive pulmonary disease. Am J Respir Crit Care Med 2004;169:1298-303.

5 Purdy S. Avoiding hospital admissions: what does the research evidence say? King's Fund, 2010.

6 Monninkhof E, van der Valk P, van der Palen J, van Herwaarden C, Partridge MR, Zielhuis G. Self-management education for patients with COPD : a systematic review. Thorax 2003;58:394-8.
7 British Thoracic Society. Burden of lung disease. 2nd ed. BTS, 2006 (available at www. brit-thoracic.org.uk/Portals/0/Library/BTS\%20Publications/burdeon of lung disease2007. pdf).

8 Royal College of Physicians, British Thoracic Society, British Lung Foundation. Report of the national chronic obstructive pulmonary disease audit 2008: clinical audit of COPD exacerbations admitted to acute NHS trusts across the UK. Royal College of Physicians, 2008.

9 Mannini DM, Buist AS. Global burden of COPD: risk factors, prevalence, and future trends. Lancet 2007:370:765-73.

10 Hodkinson HM. Evaluation of a mental test score for assessment of mental impairment in the elderly. Age Ageing 1972;1:233-8.

11 McLoone P. Carstairs scores for Scottish postcode sectors from the 2001 census. MRC Social and Public Health Sciences Unit, 2004 (available at www.sphsu.mrc.ac.uk/library/ other\%20reports/Carstairs report.pdf).

12 National Clinical Guideline Centre. Chronic obstructive pulmonary disease: management of chronic obstructive pulmonary disease in adults in primary and secondary care. National Clinical Guideline Centre, 2010. Available at http://guidance.nice.org.uk/CG101/Guidance/ pdf/English.

13 Woolhouse IS, Hill SL, Stockley RA. Symptom resolution assessed using a patient directed diary card during treatment of acute exacerbations of chronic bronchitis. Thorax 2001;56:947-53.

14 Clark NM, Gong M, Kaciroti N. A model of self-regulation for control of chronic disease. Health Educ Behav 2001;28:769-82.

15 Bourbeau J, Julien M, Maltais F, Rouleaux M, Beaupre A, Begin R, et al. Reduction of hospital utilization in patients with chronic obstructive pulmonary disease: a disease-specific self-management intervention. Arch Intern Med 2003:163:585-91.

16 Anthonisen NR, Manfreda J, Warren CPW, Hershfield ES, Harding GK, Nelson NA.Antibiotic therapy in exacerbations of COPD. Ann Intern Med 1987;106:196-204.

17 Jones PW, Quirk FH, Littlejohns P. A self-complete measure of health status for chronic airflow limitation: the St George's respiratory questionnaire. Am Rev Respir Dis 1992;145:1321-7.

18 Zigmond AS, Snaith RP. The hospital anxiety and depression scale. Acta Psychiatr Scand 1983;67:361-70.

19 Wigal JK, Cree TL, Kotses H. The COPD self-efficacy scale. Chest 1991;99:1193-6.

20 Kind, P. The EuroQol instrument: an index of health-related quality of life. In: Spilker B, ed. Quality of life and pharmacoeconomics in clinical trials. 2nd ed. Lippincott-Raven Publishers, 1996.

21 Rice KL, Dewan N, Bloomfield HE, Grill J, Schult TM, Nelson DB, et al. Disease management program for chronic obstructive pulmonary disease. Am J Respir Crit Care Med 2010;182:890-6.

22 Gonzales AZ, Suissa S, Ernst P. Gender differences in survival following hospitalisation with COPD. Thorax 2011;66:38-42.

23 Wong AWM, Gan WQ, Burns J, Sin DD, van Eeden SF. Acute exacerbation of COPD: influence of social factors in determining length of stay and readmission rates. Can Respir $J$ 2008;15:361-4.

$24 \mathrm{Xu}$ W, Collet JP, Shapiro S, Lin Y, Yang T, Platt R, et al. Independent effect of depression and anxiety on COPD exacerbations and hospitalizations. Am J Respir Crit Care Med 2008;178:913-20.

25 Lacasse Y, Goldstein R, Lasserson TJ, Martin S. Pulmonary rehabilitation for chronic obstructive pulmonary disease. Cochrane Database Syst Rev 2006;4:CD003793.

26 Silverman J, Kurtz SM, Draper J. The Calgary-Cambridge approach to communication skills teaching I: agenda-led outcome based analysis of the consultation. Education for General Practice 1996;7:288-99.

27 Silverman J, Draper J, Kurtz SM. The Calgary-Cambridge approach to communication skills teaching II: the SET-GO method of descriptive feedback. Education for General Practice 1997;8:16-23.

28 Bischoff EW, Hamd DH, Sedeno M, Schermer TR, Bernard S, Maltais F. Effects of written action plan adherence on COPD exacerbation recovery. Thorax 2011;66:26-31.

\section{Accepted: 29 December 2011}

\section{Cite this as: BMJ 2012;344:e1060}

This is an open-access article distributed under the terms of the Creative Commons Attribution Non-commercial License, which permits use, distribution, and reproduction in any medium, provided the original work is properly cited, the use is non commercial and is otherwise in compliance with the license. See: http://creativecommons.org/licenses/by$\mathrm{nc} / 2.0 /$ and http://creativecommons.org/licenses/by-nc/2.0/legalcode. 


\section{What is already known on this topic}

Acute exacerbations of chronic obstructive pulmonary disease (COPD) are debilitating for the patient and expensive for the health service

Limited self management advice is ineffective in COPD

The latest Cochrane review concluded that "supported self management" (a combination of self management training and ongoing support) shows benefit

\section{What this study adds}

No reduction in hospital admissions was seen with supported self management in COPD in the UK No differential effect in relation to factors associated with the disease itself or demographics was apparent

Subgroup analysis showed that $42 \%$ of the intervention group learnt to self manage effectively and had significantly reduced risk of readmission to hospital

\section{Tables}

\section{Table 1| Baseline characteristics of participants. Values are numbers (percentages) unless stated otherwise}

\begin{tabular}{|c|c|c|c|}
\hline Characteristic & Intervention $(\mathrm{n}=\mathbf{2 3 2})$ & Control (n-232) & All $(n=464)$ \\
\hline Mean (SD) age (years) & $70.0(9.3)$ & $68.3(9.2)$ & $69.1(9.3)$ \\
\hline Male sex & $88(38)$ & $82(35)$ & $170(37)$ \\
\hline Living alone & $92(40)$ & $96(41)$ & $188(41)$ \\
\hline Living in most deprived areas (deprivation categories 6 and 7 ) & $143(62)$ & $140(60)$ & $283(61)$ \\
\hline Pulmonary rehabilitation attendance within 2 years of study entry & $65(28)$ & $65(28)$ & $130(28)$ \\
\hline Previous hospital admission for COPD in 12 months before index admission & $135(58)$ & $137(59)$ & $272(59)$ \\
\hline Mean (SD) No of self reported prednisolone courses in previous 12 months & $4.26(3.38)$ & $4.46(3.15)$ & $4.36(3.26)$ \\
\hline Mean (SD) No of comorbidities & $2.9(2.0)$ & $2.8(1.8)$ & $2.8(1.9)$ \\
\hline Mean (SD) St George's respiratory questionnaire total score. & $70.5(16.7)$ & $69.7(16.1)$ & $70.1(16.4)$ \\
\hline Mean (SD) COPD self efficacy score & $68.2(27.5)$ & $69.8(25.5)$ & $69.0(26.5)$ \\
\hline Mean (SD) hospital anxiety and depression scale anxiety score. & $10.0(4.5)$ & $9.3(4.6)$ & $9.7(4.6)$ \\
\hline Mean (SD) hospital anxiety and depression scale depression score. & $8.5(3.9)$ & $8.3(4.1)$ & $8.4(4.0)$ \\
\hline
\end{tabular}


Table 2| Primary and secondary* outcomes

\begin{tabular}{|c|c|c|c|c|}
\hline End point (at 12 months) & Intervention $(\mathrm{n}=232)$ & Control $(n=232)$ & Treatment effect† $(95 \% \mathrm{Cl})$ & $P$ value \\
\hline No (\%) COPD admission or COPD death & $111(48)$ & $108(47)$ & 1.05 (0.80 to 1.38$)$ & 0.725 \\
\hline No (\%) COPD deaths & $23(10)$ & $16(7)$ & $1.36(0.71$ to 2.61$)$ & 0.354 \\
\hline No (\%) deaths (all causes) & $30(13)$ & $22(9)$ & 1.35 (0.77 to 2.38$)$ & 0.297 \\
\hline Mean (SD) SGRQ symptom score & $-6.01(20.85)(n=116)$ & $-4.16(22.52)(n=90)$ & $-2.17(-7.80$ to 3.46$)$ & 0.448 \\
\hline Mean (SD) SGRQ activity score & $1.44(13.27)(n=91)$ & $0.95(11.05)(n=69)$ & $0.80(-2.58$ to 4.18$)$ & 0.641 \\
\hline Mean (SD) SGRQ impacts score & $-3.16(17.12)(n=78)$ & $4.23(15.51)(n=63)$ & $-6.89(-12.40$ to -1.39$)$ & 0.015 \\
\hline Mean (SD) SGRQ total score & $-2.99(12.56)(\mathrm{n}=69)$ & $1.38(11.33)(n=53)$ & $-4.52(-9.07$ to 0.04$)$ & 0.052 \\
\hline $\begin{array}{l}\text { No (\%) with } 4 \text { point improvement in } \\
\text { SGRQ total score }\end{array}$ & $30 / 69(43)$ & $18 / 53(34)$ & $1.71(0.75$ to 3.89$)(\mathrm{OR})$ & 0.206 \\
\hline Mean (SD) CSES total score & $-1.73(34.04)(n=119)$ & $-5.55(33.72)(\mathrm{n}=94)$ & $2.65(-5.85$ to 11.14$)$ & 0.540 \\
\hline Mean (SD) HADS anxiety score & $-0.37(3.77)(n=104)$ & $0.93(3.29)(n=82)$ & $-1.06(-2.08$ to -0.03$)$ & 0.044 \\
\hline Mean (SD) HADS depression score & $0.54(3.26)(n=109)$ & $0.75(2.78)(n=84)$ & $-0.27(-1.13$ to 0.59$)$ & 0.538 \\
\hline Mean (SD) EQ-5D (area under curve) & $132.8(95.5)(n=107)$ & $139.8(100.3)(n=75)$ & $-6.9(-36.1$ to 22.4$)$ & 0.644 \\
\hline
\end{tabular}

$\mathrm{COPD}=$ chronic obstructive pulmonary disease; CSES=COPD self efficacy score; EQ-5D=EuroQol 5D; HADS=hospital anxiety and depression scale; SGRQ=St George's respiratory questionnaire.

*Numbers of paired datasets shown for each element.

†Mean differences or hazard ratios. 
Table 3| Comparison of baseline characteristics of "successful self managers" and others in intervention group. Values are numbers (percentages) unless stated otherwise

\begin{tabular}{|c|c|c|c|}
\hline Characteristic & Successful self managers $(n=75)$ & Others $(n=105)$ & $\begin{array}{l}\text { All cases completing } 12 \text { months in } \\
\text { intervention group }(n=180)\end{array}$ \\
\hline Mean (SD) age (years) ${ }^{*}$ & $67.3(8.6)$ & $71.2(9.1)$ & $69.5(9.1)$ \\
\hline Male sex & $32(43)$ & $40(38)$ & $72(40)$ \\
\hline Mean (SD) \% predicted FEV & $42.6(13.5)$ & $40.9(13.0)$ & $41.6(13.2)$ \\
\hline Living alone* & $20(27)$ & $48(46)$ & $68(38)$ \\
\hline $\begin{array}{l}\text { Living in most deprived areas (deprivation } \\
\text { categories } 6 \text { and } 7 \text { ) }\end{array}$ & $49(65)$ & $65(62)$ & $114(63)$ \\
\hline Current smokers & $25(33)$ & $44(42)$ & $69(38)$ \\
\hline $\begin{array}{l}\text { Pulmonary rehabilitation attendance within } 2 \text { years } \\
\text { of study entry }\end{array}$ & $26(35)$ & $27(26)$ & $53(29)$ \\
\hline $\begin{array}{l}\text { Hospital admission for COPD in year before study } \\
\text { entry }\end{array}$ & $40(53)$ & $54(51)$ & $94(52)$ \\
\hline $\begin{array}{l}\text { Mean (SD) self reported prednisolone courses within } \\
\text { previous year }\end{array}$ & $4.09(3.18)$ & $4.04(3.45)$ & $4.06(3.33)$ \\
\hline Mean (SD) SGRQ total score & $72.8(16.1)(n=47)$ & $68.3(18.3)(n=67)$ & $70.2(17.5)(n=114)$ \\
\hline Mean (SD) CSES total score* & $68.3(24.0)(n=65)$ & $72.7(30.2)(n=87)$ & $70.8(27.7)(n=152)$ \\
\hline Mean (SD) HADS anxiety score & $10.0(4.5)(n=62)$ & $9.5(4.4)(n=73)$ & $9.7(4.5)(n=135)$ \\
\hline Mean (SD) HADS depression score & $8.7(3.5)(n=61)$ & $7.9(4.1)(n=79)$ & $8.2(3.9)(n=140)$ \\
\hline
\end{tabular}

COPD=chronic obstructive pulmonary disease; $C S E S=C O P D$ self efficacy score; $F E V_{1}=$ forced expiratory volume in 1 second; HADS=hospital anxiety and depression scale; $S G R Q=S t$ George's respiratory questionnaire.

*Significant difference $(P<0.05)$. 
Table 4| Predictors of successful self management

Predictor Odds ratio for success* $(95 \% \mathrm{Cl}) \quad$ P value

$\begin{array}{lll}\text { Increasing age (years) } \quad 0.95(0.91 \text { to } 0.99) & 0.012\end{array}$

Living alone $0.39(0.19$ to 0.80$)$ 0.010

*Odds ratio $<1$ indicates lesser likelihood of success. 
Table 5| Healthcare outcomes, comparing successful self managers with unsuccessful self managers. Values are numbers (percentages) unless stated otherwise

\begin{tabular}{|c|c|c|c|c|}
\hline End point (at 12 months) & $\begin{array}{l}\text { Successful self managers (SSM) } \\
\qquad(\mathrm{n}=75)\end{array}$ & $\begin{array}{l}\text { Unsuccessful self managers } \\
\text { (non-SSM) }(n=105)\end{array}$ & $\begin{array}{l}\text { Hazard ratio, SSM } v \text { non-SSM (95\% } \\
\text { Cl) }\end{array}$ & $P$ value \\
\hline$\geq 1$ hospital admission due to COPD & $20(27)$ & $51(49)$ & 0.44 (0.25 to 0.76$)$ & 0.003 \\
\hline$\geq 1$ hospital admission (all causes) & $44(59)$ & $70(67)$ & 0.77 (0.52 to 1.16$)$ & 0.213 \\
\hline
\end{tabular}




\section{Figures}

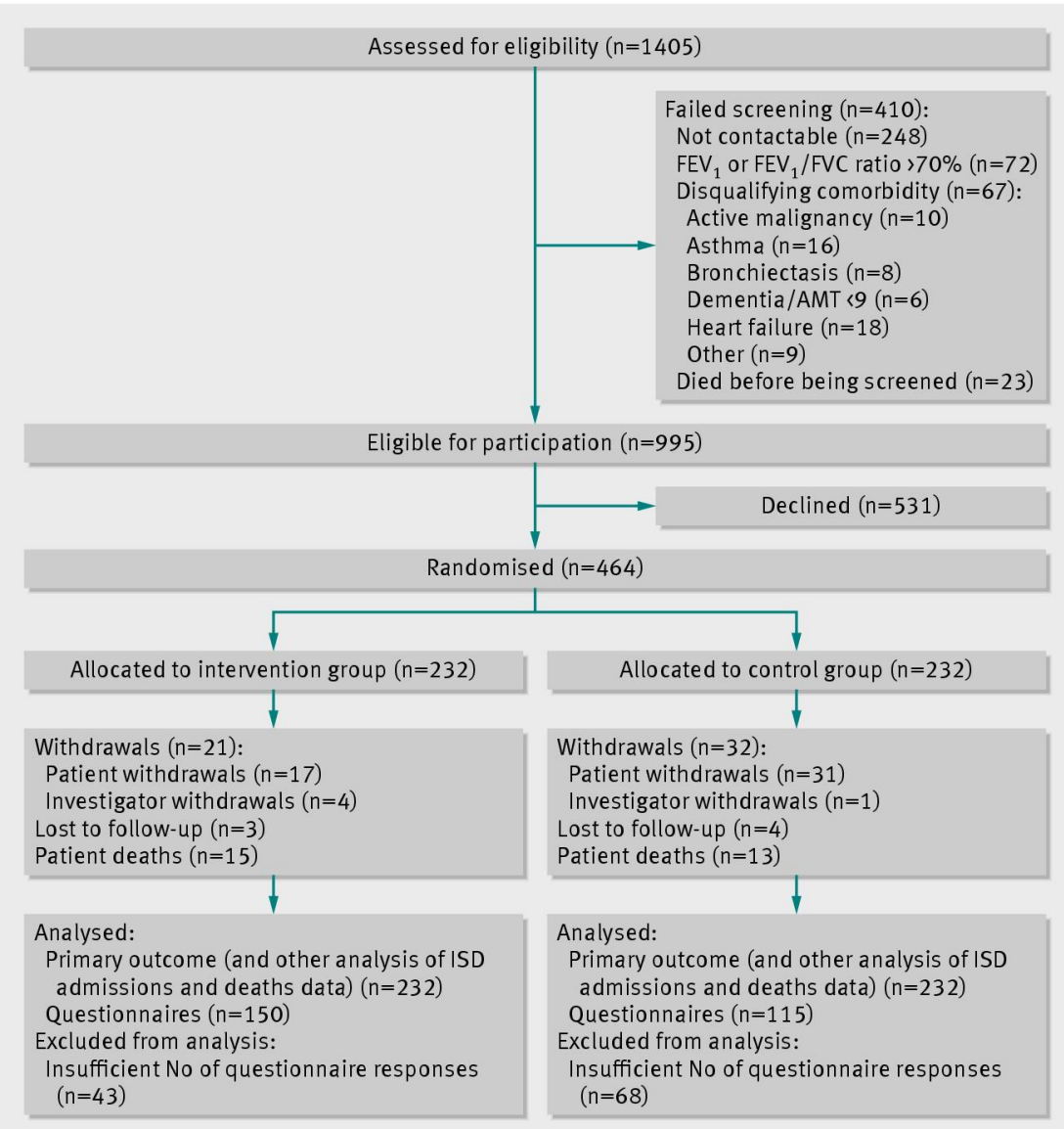

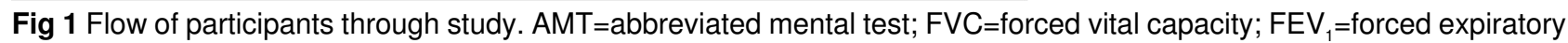
volume in 1 second; ISD=Information and Statistics Division

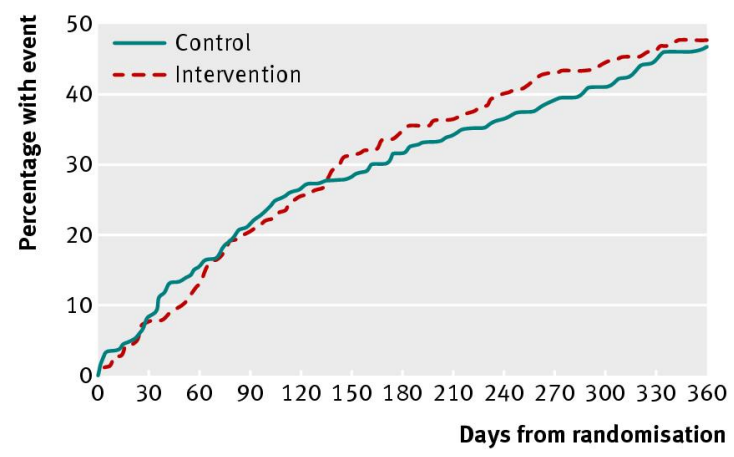

Fig 2 Hospital admissions and deaths due to chronic obstructive pulmonary disease, intervention versus control group 


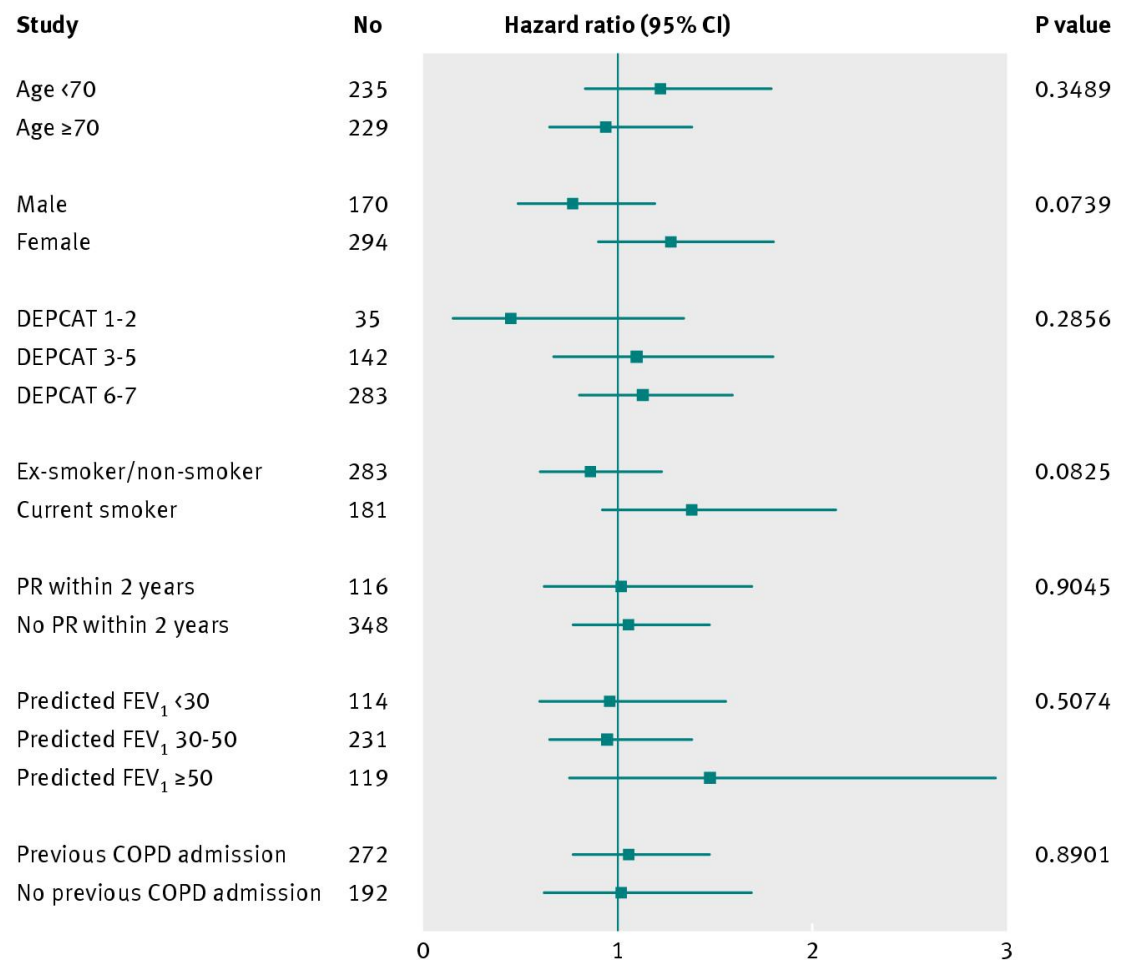

Fig 3 Subgroup analyses, intervention cohort: hazard ratios for readmission or death. COPD=chronic obstructive pulmonary disease; DEPCAT=deprivation category of area of residence ${ }^{10} ; \mathrm{FEV}_{1}=$ forced expiratory volume in 1 second; $P R=$ pulmonary rehabilitation

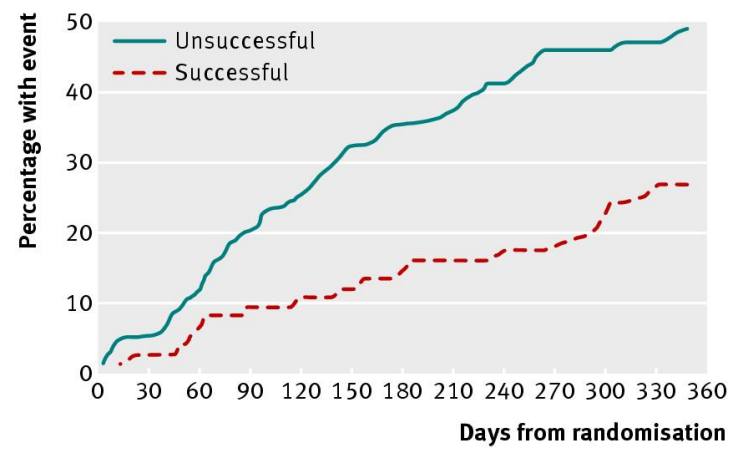

Fig 4 Readmissions for chronic obstructive pulmonary disease and deaths in successful self managers and others in intervention group 